\section{Ermächtigung des Krankenhausarztes zur Teilnahme an der vertragsärztlichen Versorgung und Sonderbedarfszulassung - geeignete Instrumente gegen den Fachärztemangel auf dem Land?}

\section{Einleitung}

Vor dem Hintergrund der fortschreitenden Urbanisierung Deutschlands sieht sich die medizinische Versorgung der Versicherten auf dem Land durch Fachärzte vor erhebliche Herausforderungen gestellt. Der „Fachärztemangel auf dem Land“ hat die gesellschaftliche Diskussion erreicht - insbesondere rurale und strukturschwache Regionen haben mit ihm zu kämpfen. Das gilt genauso für das Fachgebiet der Radiologie. Trotz rechnerischer Überversorgung in den großen Planungsbereichen, von denen Städte und gleichzeitig ländliche Regionen umfasst sind, gelingt es nicht immer, die Versorgung der Versicherten mit radiologischen Leistungen auf dem Land durch niedergelassene Fachärzte für Radiologie sicherzustellen. Während die Überversorgung in den Städten den Versorgungsgrad für den gesamten Planungsbereich anhebt und zu einer rechnerischen Überversorgung im Planungsbereich führt, bestehen in der Fläche lokal immer wieder quantitative sowie qualitative Versorgungsdefizite. Die letzten Gesundheitsreformen - das GKV-VStG von 2012 und das GKV-VSG von 2015 -, die sich des Themas angenommen haben, konnten bisher nicht zu durchgreifenden Veränderungen beitragen.

Grundsätzlich gebührt der vertragsärztlichen Versorgung der gesetzlich Versicherten durch niedergelassene Vertragsärzte und medizinische Versorgungszentren (MVZ) der Vorrang (zur Möglichkeit einer Sonderbedarfszulassung äußert sich dieser Beitrag zum Ende). Dort, wo dies nicht gelingt, kann als Instrument, um Versorgungslücken zu schließen, die Ermächtigung von Krankenhausärzten zur Teilnahme an der vertragsärztlichen Versorgung in Betracht kommen. Nach §116 S. 2 SGB V und §31a Abs. 1 S. 2 Ärzte-ZV ist eine Ermächtigung zu erteilen, soweit und solange eine ausreichende ärztliche Versorgung der Versicherten ohne die besonderen Untersu- chungs- und Behandlungsmethoden oder Kenntnisse von dafür geeigneten Krankenhausärzten nicht sichergestellt ist. Es besteht in diesen Fällen also ein Anspruch des Krankenhausarztes, zur Teilnahme an der vertragsärztlichen Versorgung ermächtigt zu werden. Gleichzeitig ergibt sich aus den Regelungen ein Vorrang der Sicherstellung der vertragsärztlichen Versorgung durch die niedergelassenen Vertragsärzte und die MVZ vor ermächtigten Krankenhausärzten. Nach aktuellen Zahlen der KBV ging zwar die Zahl ermächtigter Radiologen im Zeitraum von 2008 bis 2017 um 14,3\% zurück, dennoch verfügen weiterhin etwa $15 \%$ aller im Bundesarztregister geführten Krankenhausradiologen über eine Ermächtigung zur Teilnahme an der vertragsärztlichen Versorgung. ${ }^{1}$

Unter Einbeziehung einer aktuellen Entscheidung des Landessozialgerichts BerlinBrandenburg, ${ }^{2}$ die sich insbesondere mit dem Spannungsfeld zwischen der vertragsärztlichen Versorgung durch niedergelassene Fachärzte einerseits sowie durch ermächtigte Krankenhausärzte andererseits auseinandersetzt, werden im Folgenden zunächst die Voraussetzungen einer Ermächtigung zur Teilnahme an der vertragsärztlichen Versorgung dargestellt. Anschließend folgt eine kurze Skizzierung der Voraussetzungen einer Sonderbedarfszulassung.

\section{Ermächtigung}

Der Entscheidung des LSG lag der folgende Sachverhalt zugrunde: Der Chefarzt einer radiologischen Abteilung eines Krankenhauses hatte bei dem zuständigen Zulassungsausschuss die Verlängerung seiner bisherigen Ermächtigung zur Teilnahme an

\footnotetext{
1 http://gesundheitsdaten.kbv.de/cms/html/ 16399.php (zuletzt abgerufen am 06.08.2018).

2 LSG Berlin-Brandenburg, Urteil vom 07.09.2017, Az.: L 24 KA 54/16.
}

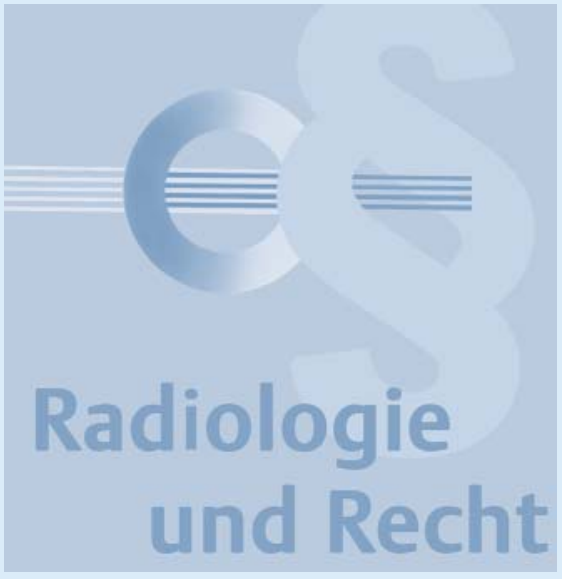

der vertragsärztlichen Versorgung beantragt. Diese umfasste bereits die Erbringung konventioneller Röntgendiagnostik auf Überweisung eines niedergelassenen Chirurgen. Neben der beantragten Verlängerung begehrte er eine Erweiterung seiner Ermächtigung zur Erbringung konventioneller Röntgendiagnostik auch auf Überweisung einer niedergelassenen Fachärztin für Orthopädie und Unfallchirurgie. Zur Begründung führte er an, dass deren möglichen Unfallpatienten ein Transport zu den nächsten niedergelassenen Radiologen im zehn Kilometer entfernten Nachbarort nicht zuzumuten sei. Der Zulassungsausschuss beschied den Antrag auf Verlängerung sowie den Erweiterungsantrag zunächst positiv. Hinsichtlich der Erweiterung der Ermächtigung legte jedoch die radiologische Berufsausübungsgemeinschaft aus dem zehn Kilometer entfernten Nachbarort Drittwiderspruch ein. Begründend führte sie an, dass eine Ermächtigung zum Tätigwerden auf Überweisung der Fachärztin nicht erforderlich sei, da deren Leistungen im Rahmen ihrer orthopädischen Ambulanz planbar durch niedergelassene Radiologen durchführbar seien, ohne die ausreichende Versorgung der Versicherten zu gefährden. Unter Anschluss an diese Begründung half der Berufungsausschuss dem Widerspruch ab. Der Chefarzt begehrte nun im Klageweg zunächst vor dem zuständigen Sozialgericht Potsdam die Ermächtigung zur Teilnahme an der vertragsärztlichen Versorgung auch auf Überweisung durch die niedergelassene Fachärztin für Orthopädie und Unfallchirurgie. ${ }^{3}$ Sowohl das SG als auch das LSG verneinen das Vorliegen der

3 SG Potsdam, Urteil vom 28.09.2016, Az.: S 1 KA 100/15. 
Voraussetzungen einer Erweiterung der Ermächtigung des Chefarztes.

\section{Voraussetzungen der Ermächtigung}

\section{Antrag - persönliche Voraussetzun- gen - Zustimmung des Arbeitgebers}

Eine Ermächtigung zur Teilnahme an der vertragsärztlichen Versorgung wird nach $\S 31$ a Abs. 2 S. 1 Ärzte-ZV nur auf Antrag des Krankenhausarztes erteilt. In dem Antrag sind die vertragsärztlichen Leistungen, für die die Ermächtigung erteilt werden soll, im Einzelnen anzugeben. Der Antragsteller muss die persönlichen Voraussetzungen einer Ermächtigung zur Teilnahme an der vertragsärztlichen Versorgung erfüllen. Er muss in einem Beschäftigungsverhältnis zu dem Rechtsträger eines Krankenhauses, einer Vorsorge- oder Rehabilitationseinrichtung oder einer stationären Pflegeeinrichtung stehen sowie eine abgeschlossene Weiterbildung zum Facharzt nachweisen können (§116 S. 1 SGB V, §31a Abs. 1 S. 1 Ärzte-ZV). Zudem darf nur ein Arzt ermächtigt werden, der im Sinne des § 21 Ärzte-ZV zur Teilnahme an der vertragsärztlichen Versorgung nicht als ungeeignet erscheint ( $\S 31$ a Abs. 3 i. V. m. $\S 31$ Abs. 8 Ärzte-ZV). Eine Ermächtigung ist schließlich nur mit Zustimmung des Rechtsträgers der Einrichtung, in der der Arzt tätig ist, möglich (§116 S. 1 SGB V, $\S 31$ a Abs. 1 S. 1 Ärzte-ZV).

\section{Ermächtigungsbedarf}

Die Ermächtigung ist trotz des Vorrangs der Versorgung durch zugelassene Vertragsärzte oder MVZ jedoch nur zu erteilen, wenn ein Ermächtigungsbedarf besteht, weil eine ausreichende Versorgung der Versicherten ohne die besonderen Untersuchungs- und Behandlungsmethoden oder Kenntnisse entsprechend geeigneter Krankenhausärzte nicht sichergestellt ist (vgl. §116 S. 2 SGB V, §31a Abs. 1 S. 2 Ärzte-ZV).

Dabei kommt den Zulassungsgremien hinsichtlich des Tatbestandmerkmals der nicht sichergestellten ärztlichen Versorgung als unbestimmtem Rechtsbegriff ein gerichtlich nur eingeschränkt überprüfbarer Beurteilungsspielraum zu. Die gerichtliche
Prüfung ist deshalb reduziert auf die Einhaltung eines fairen Verfahrens, die Richtigkeit und Vollständigkeit der Ermittlung des Sachverhaltes, die Einhaltung der Grenzen des durch Auslegung zu ermittelnden Inhalts des unbestimmten Rechtsbegriffs und die Nachvollziehbarkeit der Begründungserwägungen. ${ }^{4}$ Maßgebend für die Beurteilung ist, abgesehen von einzelnen Ausnahmen, nur der vollständige Planungsbereich, in dem der Leistungsort der $\mathrm{Er}$ mächtigung gelegen ist. Zur Prüfung des Bedarfs können die Zulassungsgremien bspw. die weiteren Leistungserbringer im Planungsbereich befragen und die Angaben über Leistungsstatistiken auf Plausibilität überprüfen. Einzubeziehen sind sämtliche Leistungsangebote, die gegenüber der $\mathrm{Er}$ mächtigung nicht nachrangig sind.

Ein Ermächtigungsbedarf kann in der Form eines quantitativ-allgemeinen oder eines qualitativ-speziellen Bedarfs vorliegen.

\section{Quantitativ-allgemeiner Bedarf}

Ein quantitativ-allgemeiner Bedarf besteht, wenn in dem jeweiligen Planungsbereich eine zu geringe Zahl an Vertragsärzten mit einer zur Deckung eines bestimmten Bedarfs erforderlichen Qualifikation ansässig ist und so eine ausreichende und zweckmäßige Versorgung der Versicherten nicht zu gewährleisten ist. Dabei ist die Erteilung einer Ermächtigung aus quantitativ-allgemeinen Gründen regelmäßig ausgeschlossen, wenn für die betreffende Arztgruppe Überversorgung i. S. v. § 103 Abs. 1 SGB V i. V. m. $\S 16 b$ Abs. 2 Ärzte-ZV festgestellt ist. ${ }^{5}$

In dem von dem LSG entschiedenen Fall betrug der Versorgungsgrad im Planungsbereich $176,8 \%$. Entsprechend bestanden für das LSG keine Gründe, die Beurteilung des Berufungsausschusses, dass ein quantitativ-allgemeiner Bedarf nicht bestehe, in Zweifel zu ziehen.

\section{Qualitativ-spezieller Bedarf}

Ein qualitativ-spezieller Bedarf liegt hingegen vor, wenn im betreffenden Planungsbereich zwar absolut eine ausreichende An-

4 vgl. BSG, Urteil vom 12.09.2001, Az.: B 6 KA $86 / 00$.

5 BSG, Urteil vom 19.07.2006, Az.: B 6 KA 14/ $05 \mathrm{R}$ m.w. N. zahl zugelassener Fachärzte ansässig ist, diese jedoch ein spezielles Leistungsangebot in Form bestimmter Behandlungsoder Untersuchungsmethoden nicht oder nicht in ausreichendem Maße anbieten und daraus resultierend eine ausreichende und zweckmäßige Versorgung der Versicherten nicht gewährleisten können. Eine Ermächtigung im Rahmen eines qualitativspeziellen Bedarfs setzt deshalb voraus, dass der zu ermächtigende Krankenhausarzt über besondere Kenntnisse und Fähigkeiten oder Behandlungs- bzw. Untersuchungsmethoden verfügt, die sich in seinem Leistungsangebot dergestalt niederschlagen, dass dieses geeignet ist, den qualitativ-speziellen Versorgungsbedarf zu decken, sowie gleichzeitig für eine ausreichende medizinische Versorgung erforderlich ist. ${ }^{6}$

Hinsichtlich eines qualitativ-speziellen Versorgungsbedarfs hat sich das LSG in der von ihm entschiedenen Angelegenheit den Wertungen des Berufungsausschusses im Ergebnis angeschlossen und einen solchen bejaht. Allerdings stellt sich die Frage, ob die Feststellungen des Berufungsausschusses wegen eines Überschreitens des Beurteilungsspielraums nicht einer gerichtlichen Prüfung hätten unterzogen werden müssen.

Das LSG hat lediglich festgestellt, dass die Entscheidung des Berufungsausschusses angesichts des ihm eingeräumten Beurteilungsspielraums nicht zu beanstanden sei. Die Feststellungen des Berufungsausschusses, dass die Leistungen in einer orthopädischen Praxis grundsätzlich eher planbar seien als in einer chirurgischen Praxis und dass eine Fachärztin für Orthopädie und Unfallchirurgie nur selten und unregelmäßig Patienten behandelt, bei denen eine sofortige radiologische Behandlung aus medizinischen Gründen geboten erscheint, sind von dem LSG pauschal als nachvollziehbar bewertet worden. Es hat die Bewertung des Berufungsausschusses, dass es für die Sicherstellung der vertragsärztlichen Versorgung ausreichend ist, wenn die Patienten der niedergelassenen Fachärztin für Orthopädie und Unfallchirurgie die radiologische Berufsausübungsgemeinschaft in zehn Kilometern Entfernung aufsuchen, deshalb bestätiat. Unbeachtet aeblieben

6 BSG, Urteil vom 14.07.1993, Az.: 6 RKa 71/91. 
sind bei diesen Wertungen, dass es für die Einhaltung der Grenzen des den Zulassungsgremien eingeräumten Beurteilungsspielraums einer detaillierteren Prüfung der ausreichenden Versorgung der Versicherten bedurft hätte. Unter Heranziehung und Würdigung der (Muster-)Weiterbildungsordnung hätte es nahegelegen zu prüfen, ob durch eine niedergelassene Fachärztin für Orthopädie und Unfallchirurgie tatsächlich kaum Unfallpatienten mit nicht planbaren Untersuchungen überwiesen werden. Dabei hätte allein die Bezeichnung „Unfallchirurgie“ nahegelegt, dass die niedergelassene Fachärztin auch Patienten mit Unfallverletzungen versorgt, deren Behandlungen nicht langfristig planbar sind.

Unabhängig von der Frage, ob diese pauschale Wertung des LSG letztlich ausreichend war, sind seine anschließenden Ausführungen, dass in Notfällen eine Leistungserbringung durch den Chefarzt zugunsten der Versicherten und zulasten der gesetzlichen Krankenversicherung nach $\S 76$ Abs. 1 S. 2 SGB V möglich wäre, äußerst fragwürdig. Durch $\S 76$ Abs. 1 S. 2 SGB V soll nach der Rechtsprechung des BSG gerade kein „zweiter Versorgungsweg“ eröffnet werden. ${ }^{7}$ Folgte man jedoch den Ausführungen des LSG, müsste den Versicherten ermöglicht werden, die Notfallambulanzen der Krankenhäuser zu jeder Zeit allein aufgrund räumlicher Nähe aufzusuchen. Dies liefe den Bestrebungen des Gesetzgebers, die Inanspruchnahme der Notfallambulanzen der Krankenhäuser einzudämmen, entgegen.

Anhand der Entscheidung des LSG wird aber deutlich, dass die Bewertung, ob ein qualitativ-spezieller Ermächtigungsbedarf vorliegt, eben nicht aufgrund pauschaler Erwägungen erfolgen darf, sondern dass eine effektive gerichtliche Kontrolle der Entscheidungen der Zulassungsgremien möglich sein muss, wenn diese den ihnen eingeräumten Beurteilungsspielraum überschreiten. Insbesondere darf der Vorrang der Zulassung vor der Ermächtigung nicht so weit gehen, dass die Sicherstellung der vertragsärztlichen Versorgung der Versicherten gefährdet wird und dass für die Versicherten der Weg in die Notfallambu-

7 BSG, Urteil vom 02.07.2014, Az.: B 6 KA 30/ $13 R$. lanzen der Krankenhäuser faktisch zu einem generellen Versorgungsweg wird. In vielen ländlichen Regionen mit einem Mangel an Fachärzten für Radiologie wird für Krankenhausradiologen trotz einer rechnerischen Überversorgung im Planungsbereich die Möglichkeit bestehen, aufgrund des Vorliegens eines qualitativ-speziellen Ermächtigungsbedarfs einen erfolgreichen Antrag für eine Ermächtigung zur Erbringung besonderer vertragsärztlicher Leistungen zu stellen. Dabei ist das Instrument der Ermächtigung inzwischen nicht mehr nur Chefärzten vorbehalten, sondern auch für nachgeordnete Fachärzte eröffnet. Voraussetzung ist die hauptberufliche ärztliche Tätigkeit im Krankenhaus, sodass die Teilnahme an der vertragsärztlichen Versorgung nicht im Mittelpunkt der ärztlichen Tätigkeit des Arztes steht. ${ }^{8}$

\section{Vor- und Nachteile der Ermächtigung}

Mit der Ermächtigung von Krankenhausärzten zur Teilnahme an der vertragsärztlichen Versorgung können nicht nur die Kassenärztlichen Vereinigungen ihren Sicherstellungsauftrag erfüllen, sondern zudem die Notfallambulanzen der Krankenhäuser entlastet werden.

Zugleich ist die Ermächtigung eines Krankenhausarztes immer auch eine Ausnahme. Dies kommt zum einen dadurch zum Ausdruck, dass sie zeitlich und räumlich sowie ihrem Umfang nach zu begrenzen ist ( §§31a Abs. 3, 31 Abs. 7 S. 1 ÄrzteZV). Das bedeutet, dass sie zu befristen ist und zudem auf Patienten aus einem begrenzten räumlichen Umkreis beschränkt werden kann. Außerdem sind die erbringbaren Leistungen zu bezeichnen, da die Ermächtigung, anders als die Zulassung, nur für die Leistungen erteilt wird, für die ein Bedarf besteht. Zudem ist in dem Ermächtigungsbeschluss auszusprechen, ob der Arzt unmittelbar oder nur auf Überweisung (bestimmter Ärzte) in Anspruch genommen werden darf. In dem entschiedenen Fall geschah dies durch ausdrückliche Bezeichnung des niedergelassenen Facharztes auf dessen Überweisung der Chefarzt tätig werden darf, sowie durch die Bezeichnung

8 BSG, Urteil vom 20.03.2013, Az.: B 6 KA 26/ $12 \mathrm{R}$. der Gebührenordnungspositionen des EBM, die er zulasten der Kassenärztlichen Verneinung zur Abrechnung bringen darf. Zum anderen wird die Abrechnung der Leistungen eines ermächtigten Arztes nach § 8 Abs. 4 der Richtlinien zum Inhalt und zur Durchführung der Prüfungen gemäß $\S 106$ d Abs. 6 SGB V (Abrechnungsprüfungs-Richtlinien) bereits dann auffällig, wenn unter Zugrundelegung der Prüfzeiten nach Anhang 3 zum Einheitlichen Bewertungsmaßstab lediglich mehr als 156 Stunden im Quartalsprofil erreicht werden. Darüber hinaus hat der ermächtigte Arzt sämtliche ärztlichen Leistungen höchstpersönlich zu erbringen, sodass eine Delegation ärztlicher Leistungen im Rahmen einer Ermächtigung ausgeschlossen ist.

\section{Sonderbedarfszulassung als Alternative}

Eine Alternative zur Ermächtigung ist die Sonderbedarfszulassung nach $\S 101$ Abs. 1 S. 1 Nr. 3 SGB V i. V.m §§36, 37 Bedarfsplanungs-Richtlinie (BPIRL). Diese wird ebenfalls bei Vorliegen eines lokalen oder qualifikationsbezogenen Sonderbedarfs erteilt. Zudem müssen die allgemeinen Zulassungsvoraussetzungen erfüllt sein.

\section{Vorrangige Erfüllung des vertrags- ärztlichen Versorgungsauftrags}

Zu diesen allgemeinen Zulassungsvoraussetzungen zählt nach $\S 32$ Abs. 1 S. 1 ÄrzteZV, dass die vertragsärztliche Tätigkeit persönlich und in freier Praxis ausgeübt wird. Voraussetzung dafür ist, dass die berufliche und persönliche Selbständigkeit des Arztes gesichert ist und erhebliche Einflussnahmen Dritter ausgeschlossen sind. Dass der Vertragsarzt das wirtschaftliche Risiko seiner Praxis trägt und an deren wirtschaftlichem Erfolg beteiligt ist, gehört dabei zur eigenverantwortlichen Gestaltung der ärztlichen Tätigkeit. Die Tätigkeit im Rahmen einer Sonderbedarfszulassung setzt deshalb voraus, dass der Arzt freiberuflich in seiner eigenen Praxis tätig wird, während die Ermächtigung eines Krankenhausarztes im Rahmen des bestehenden Anstellungsverhältnisses zum Krankenhaus erteilt werden kann. Weil § 20 Abs. 1 Ärzte-ZV zudem verlangt, dass der Zulassung zur vertragsärztlichen Versorgung kein anderes Beschäfti- 
gungsverhältnis und keine andere nicht ehrenamtliche Tätigkeit entgegenstehen, muss der Krankenausarzt seine Tätigkeit entweder vollständig in die Freiberuflichkeit verlagern oder mit seinem Arbeitgeber vertragliche Regelungen treffen, die ihm die Einrichtung seiner eigenen Praxis ermöglichen. Dafür wird er den Arbeitsvertrag mit seinem Arbeitgeber anpassen bei einer vollzeitigen Tätigkeit insbesondere die vertragliche Arbeitszeit reduzieren und weitere Verträge, z. B. einen Kooperationsvertrag und einen Mietvertrag über Praxisräume, einen Vertrag zur Nutzungsüberlassung der radiologischen Geräte sowie einen Leistungserbringungsvertrag schließen müssen. Zudem wird er, wenn auch in geringem Umfang, eigenes Personal in seiner neuen Praxis anstellen oder einen Vertrag zur Personalgestellung mit seinem Arbeitgeber abschließen müssen.

Die parallele Tätigkeit als angestellter Arzt im Krankenhaus und als Arzt in freier Praxis mit Sonderbedarfszulassung dürfte dabei künftig erschwert werden, da aufgrund aktueller Bestrebungen des Gesetzgebers neben der Erfüllung des Versorgungsauftrags kaum noch planbare Zeit für die Tätigkeit als Krankenhausarzt vorhanden sein wird. Nach dem aktuellen Referentenentwurf des BMG über das Terminservice- und Versorgungsgesetz (TSVG) ${ }^{9}$ sollen die Mindestsprechstundenzeiten für Vertragsärzte mit einem vollen Versorgungsauftrag von 20 auf 25 Stunden erhöht werden (vgl. §17 Abs. 1a S. 1 BMV-Ä). Bereits nach der geltenden Rechtslage ist nach Ansicht des BSG jedoch zu den mindestens 20 Sprechstunden pro Woche ein Aufschlag von 30 bis 50 \% für notwendige Begleitleistungen zu addieren, ${ }^{10}$ sodass sich derzeit für den vollen Versorgungsauftrag eine Zeit von insgesamt mindestens 26 bis 30 wöchentlichen Arbeitsstunden als Vertragsarzt ermitteln lässt.

9 abrufbar unter: https://www.bundesgesundheitsministerium.de/fileadmin/Dateien/ 3_Downloads/ Gesetze_und_Verordnungen/GuV/T/ TSVG_RefE.pdf (zuletzt abgerufen am 06.08.2018).

10 BSG, Urteil vom 13.10.2010, Az.: B 6 KA 40 $09 R$.
Rechnet man den vom BSG geforderten Aufschlag von 30 bis $50 \%$ für notwendige Begleitleistungen für den vollen Versorgungsauftrag nach einer Anhebung der wöchentlichen Mindestsprechstundenzeit auf 25 Stunden hinzu, müssten niedergelassene Radiologen zukünftig mindestens 32,5 bis 37,5 Stunden wöchentlich für die Tätigkeit als Vertragsarzt zur Verfügung stehen. Die Möglichkeit der Versorgung von Krankenhauspatienten würde dadurch zeitlich erheblich eingeschränkt, zumal die Sprechstundenzeiten zu den üblichen Praxiszeiten anzubieten sind.

Um zu verhindern, dass sich die Arbeitszeiten eines Krankenhausradiologen aufgrund der Erhöhung der Sprechstundenzeiten im Rahmen seines Anstellungsverhältnisses künftig reduzieren müssen, bestünde die Möglichkeit, die Sonderbedarfszulassung auf die Hälfte zu reduzieren. Wie bei einer regulären Zulassung kann auch eine Sonderbedarfszulassung mit einem hälftigen Versorgungsauftrag wahrgenommen werden.

\section{Sonderbedarfsfeststellung}

Als besondere Zulassungsvoraussetzung muss bei der Beantragung einer Sonderbedarfszulassung ein zusätzlicher lokaler oder ein qualifikationsbezogener Sonderbedarf vorliegen.

Gemeinsame Voraussetzungen sind, dass die beantragte Sonderbedarfszulassung auf die vertragsärztliche Krankenbehandlung ausgerichtet ist und dass der Antragsteller in der Lage ist, an der Bedarfsdeckung mitzuwirken, d. h., dass er insbesondere über die notwendige medizinisch-technische Ausstattung und die erforderlichen persönlichen Qualifikationen verfügen muss. Zudem muss sich die beantragte Sonderbedarfszulassung auf eine Region beziehen, die vom projektierten Praxisstandort aus versorgt werden soll (BPIRL), und der Standort muss für die beantragte Versorgung geeignet sein (§36 Abs. 3 Nr. 2 BPIRL). Schließlich schreibt $\S 36$ Abs. 5 BPIRL vor, dass der Sonderbedarf dauerhaft erscheinen muss. Bei nur vorübergehendem Bedarf ist von der zu befristenden Ermächtigung Gebrauch zu machen.

\section{Lokaler Sonderbedarf}

Ein zusätzlicher lokaler Sonderbedarf ist anzunehmen, wenn die ausnahmsweise Besetzung eines zusätzlichen Vertragsarztsitzes unerlässlich ist, um die vertragsärztliche Versorgung in einem Versorgungsbereich zu gewährleisten und dabei einen zusätzlichen Versorgungsbedarf zu decken, und wenn im Übrigen die sonstigen Voraussetzungen des $\S 36$ BPIRL vorliegen.

Nach §36 Abs. 4 S. 3 BPIRL ist es Voraussetzung für eine Sonderbedarfszulassung wegen eines zusätzlichen lokalen Sonderbedarfs, dass aufgrund von Besonderheiten des maßgeblichen Planungsbereichs (z. B. Struktur, Zuschnitt, Lage, Infrastruktur, geografische Besonderheiten, Verkehrsanbindung, Verteilung der niedergelassenen Ärzte) ein zumutbarer Zugang der Versicherten zur vertragsärztlichen Versorgung nicht gewährleistet ist und aufgrund dessen Versorgungsdefizite bestehen. Damit ist eine in Bezug auf die Bedürfnisse der Versicherten im Planungsbereich unausgewogene Verteilung der an sich rechnerisch ausreichend zugelassenen Fachärzte der betreffenden Arztgruppe gemeint. Anders als bei der Ermächtigung ist in die Betrachtung deshalb nicht der vollständige Planungsbereich, sondern der lokale Versorgungsbedarf am Standort der (projektierten) Praxis einzubeziehen.

\section{Qualifikationsbezogener Sonderbedarf}

Ein qualifikationsbezogener Sonderbedarf ist anzunehmen, wenn die ärztlichen Tätigkeiten des qualifizierenden Inhalts in dem betreffenden Planungsbereich von zugelassenen Vertragsärzten nicht oder nicht ausreichend angeboten werden und der Arzt die für den besonderen Versorgungsbereich erforderlichen Qualifikationen nachweist.

Die besondere Qualifikation des Arztes ist in dem Umfang anzunehmen, wie sie durch den Inhalt des Schwerpunktes einer fakultativen Weiterbildung oder einer besonderen Fachkunde beschrieben wird (vgl. §37 Abs. 2 BPIRL). Nach $\S 37$ Abs. 1 lit. b), Abs. 3 BPIRL muss die Prüfung und Feststellung eines qualifikationsbezogenen Sonderbedarfs den besonderen Qualifikationen des Arztes entsprechen, wobei die Subspeziali- 
sierung Leistungen umfassen muss, die die gesamte Breite des spezialisierten Versorgungsbereichs ausfüllen.

\section{Vor- und Nachteile der Sonderbedarfszulassung}

Der Vorteil einer Sonderbedarfszulassung besteht zweifelsohne darin, dass sie, anders als eine Ermächtigung, zeitlich unbefristet erteilt wird und gegenüber einer Ermächtigung vorrangig zu erteilen ist. Zudem besteht bei der Anstellung von Ärzten die Möglichkeit der Delegation ärztlicher Leistungen. Im Rahmen der Abrechnung gelten darüber hinaus nicht die strengen Maßstäbe wie bei einer Ermächtigung.

Eine Sonderbedarfszulassung setzt aber den Schritt des Arztes in die Freiberuflichkeit und vielfältige Vertragsabschlüsse sowie die Bereitschaft des Arztes voraus, das jeweils damit verbundene wirtschaftliche Risiko zu tragen. Die Möglichkeit, neben einer Sonderbedarfszulassung angestellt tätig zu sein, wird durch die Erhöhung der Mindestsprechstundenzeiten für Vertragsärzte durch das TSVG künftig voraussichtlich weiter eingeschränkt werden. Zu beachten ist zudem, dass eine Sonderbedarfszulassung trotz ihrer höheren Voraussetzungen und der Notwendigkeit der Einrichtung einer eigenen Praxis - ebenso wie die Ermächtigung - nur bedingt verlegt und nicht nachbesetzt werden kann. §36
Abs. 7 BPIRL schreibt insoweit vor, dass es auch bei unverändertem Vorliegen der Voraussetzungen für eine Sonderbedarfszulassung im Falle einer Nachfolgebesetzung nach § 103 Abs. 4 SGB V der erneuten Zulassung bedarf und dass der Fortbestand der Sonderbedarfsfeststellungen nur mit Festsetzung einer erneuten Beschränkung erteilt werden kann. Zudem finden die Regelungen in $\S 103$ Abs. 3a S. 3 zweiter $\mathrm{Hs}$. (Nachbesetzung durch privilegierte Personenkreise) und S. 8 (Entschädigung) SGB $\mathrm{V}$ keine Anwendung. Eine wirtschaftliche Verwertung der Zulassung bei einer Beendigung der Tätigkeit oder einem Ortswechsel kommt daher auch bei der Sonderbedarfszulassung nur sehr eingeschränkt in Betracht.

\section{Fazit}

Sowohl die Ermächtigung eines Krankenhausarztes zur Teilnahme an der vertragsärztlichen Versorgung als auch eine Sonderbedarfszulassung können geeignet sein, um dem Fachärztemangel auf dem Land zu begegnen. In rechnerisch überversorgten Planungsbereichen eröffnen sie die Möglichkeit, außerhalb der Bedarfsplanung einen besonderen lokalen und qualitativen Versorgungsbedarf zu decken. Dabei stellt insbesondere die Ermächtigung einen verhältnismäßig unaufwendigen Weg dar, einem
Krankenhausarzt die Teilnahme an der vertragsärztlichen Versorgung zu ermöglichen. Zwar wird eine Ermächtigung zur Teilnahme an der vertragsärztlichen Versorgung nur befristet und mit zusätzlichen Bestimmungen hinsichtlich der abrechenbaren Leistungen erteilt, jedoch kann der ermächtigte Krankenhausarzt seine Tätigkeit als angestellter Arzt im Krankenhaus unverändert ausüben und muss das wirtschaftliche Risiko einer freiberuflichen Tätigkeit nicht eingehen. Gerade das Risiko der Freiberuflichkeit und die unsicheren Erlösmöglichkeiten auf dem Land sind es doch oftmals, die einen Fachärztemangel auf dem Land mitbedingen.

Prof. Dr. Peter Wigge

Rechtsanwalt

Fachanwalt für Medizinrecht

Jonas Kaufhold

Rechtsanwalt

Florian Markus Dawe

Wissenschaftlicher Mitarbeiter

Rechtsanwälte Wigge

Scharnhorststraße 40

48151 Münster

Telefon: (0251) $53595-0$

Telefax: (0251) 53595 - 99

E-mail: kanzlei@ra-wigge.de

www.ra-wigge.de 